\title{
Numerical Analysis of Sound in the Main Steam Stop Valve, and a Sound Suppression Method*
}

\author{
Akinori TAMURA**, Shiro TAKAHASHI**, Shunichi SATO*** \\ and Shigekazu HORI*** \\ ${ }^{* *}$ Hitachi Research Laboratory, Hitachi, Ltd. \\ 7-2-1, Omika, Hitachi, Ibaraki, Japan \\ E-mail: akinori.tamura.mt@hitachi.com

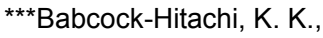 \\ 6-9, Takaramachi, Kure, Hiroshima, Japan
}

\begin{abstract}
The main steam stop valve (MSV) in thermal or nuclear power plants is liable to undergo excitation by sound having a specific frequency. The purposes of this article are to clarify the mechanism of the sound generation in the MSV using numerical analysis and to propose a suppression method for the sound generation. The analysis results were validated experimentally using a $1 / 5$-scale model of a typical MSV. The analysis results showed good agreement with the experimental data. From the analysis results, it was clarified that the sound of the MSV was generated by the hole-tone in the opening and the acoustic resonance in the vertical direction of the MSV. A method was proposed to install triangular or slanted tabs at the inlet seat of the MSV in order to prevent formation of vortex rings in the opening which leads to the hole-tone. Effects of the proposed method were tested experimentally. Eight triangular tabs having a height of $15 \%$ of the inner diameter in the seat reduced the peak value of the sound pressure by $60 \%$. Eight slanted tabs having a height of $7.5 \%$ of the inner diameter in the seat suppressed generation of the hole-tone in the MSV. These results showed that the slanted tabs are effective in suppression of the hole-tone compared with the triangular tabs.
\end{abstract}

Key words: Main Steam Stop Valve, Flow-Acoustic Interaction, Sound, Hole-Tone, Finite Difference Lattice Boltzmann Method, Suppression Method

\section{Introduction}

The main steam stop valve (MSV) is installed in the main steam lines of thermal or nuclear power plants, in order to block the main steam flowing into the turbine when it is off-line. The gate valve shown in Fig. 1 is often used as the MSV. The gate valve is composed of a valve body, seat, throats, opening, a disk to block steam, and a bonnet to accommodate the disk. During plant operation, the disk is held in place in the bonnet as shown in Fig. 1. The MSV consisting of the gate valve is liable to undergo excitation by sound having a specific frequency during plant operation. This phenomenon is undesirable because the induced sound causes loud noise and vibration of mechanical structures. From an engineering viewpoint, it is important to clarify the mechanism of sound generation and to propose a suppression method for the sound generation.

In flow fields having openings and edges, a self-sustained sound called the hole-tone often occurs. ${ }^{(1-6)}$ When a jet, issued from a nozzle, passes through a hole in a plate, placed a little downstream from the nozzle, the shear layer of the jet is unstable and rolls up into a large vortex ring. This ring cannot pass through the hole in the plate and hits the edge of the 
hole, where it generates an acoustic wave. The acoustic wave is thrown back to the nozzle, where it disturbs the shear layer. This initiates the roll-up of a new vortex ring. In this way, a self-sustained sound is formed by a flow-acoustic interaction. ${ }^{(5)}$ The MSV consisting of the gate valve has an opening and edges such as the bonnet and the seat. It is likely that the hole-tone causes the MSV to undergo excitation by the sound. However, it is unclear whether the hole-tone actually occurs in a complex geometry such as the MSV.

A numerical analysis is helpful to predict occurrence of flow-acoustic interactions and also to provide easy understanding of phenomena in the MSV. However, a numerical analysis of flow-acoustic interactions such as the hole-tone is difficult due to the need to capture the interaction between the vortex and the acoustic wave in the flow field. In particular, pressure fluctuations of the acoustic wave are very small compared with those of the vortex when flow-acoustic interactions begin to occur. Therefore, the numerical analysis method which can accurately capture the acoustic wave is required for flow-acoustic simulations. The compact finite difference scheme $e^{(6)-(11)}$ is often applied to computational aero-acoustics (CAA) simulations. Matsuura and Nakano ${ }^{(6)}$ successfully simulated the hole-tone feedback system at very low Mach number using the 6th-order tridiagonal compact scheme. However, the compact finite difference scheme is liable to become unstable and requires large computational effort compared with conventional finite difference schemes due to its implicit algorithms. ${ }^{(12)}$ Recently, the finite difference lattice Boltzmann method (FDLBM) $)^{(13)-(22)}$ has been verified as an alternative method which can accurately capture the acoustic wave. The FDLBM is the numerical analysis method that developed from the lattice Boltzmann method (LBM). ${ }^{(23)-(29)}$ Unlike conventional computational fluid dynamics (CFD) methods based on discretizations of the fluid dynamics equations, the LBM is based on the kinetic equation of the molecular gas dynamics. The FDLBM in which the kinetic equation is discretized by the finite difference scheme has been developed to improve numerical stability of the LBM.

The purposes of this article are to clarify the mechanism of sound generation in the MSV using numerical analysis and to propose a suppression method of the sound generation. As the numerical analysis method, we apply the FDLBM which has been computationally accelerated. The numerical results are validated experimentally in which 1/5-scale model of a typical MSV was used. We also experimentally test the suppression method of the sound generation.

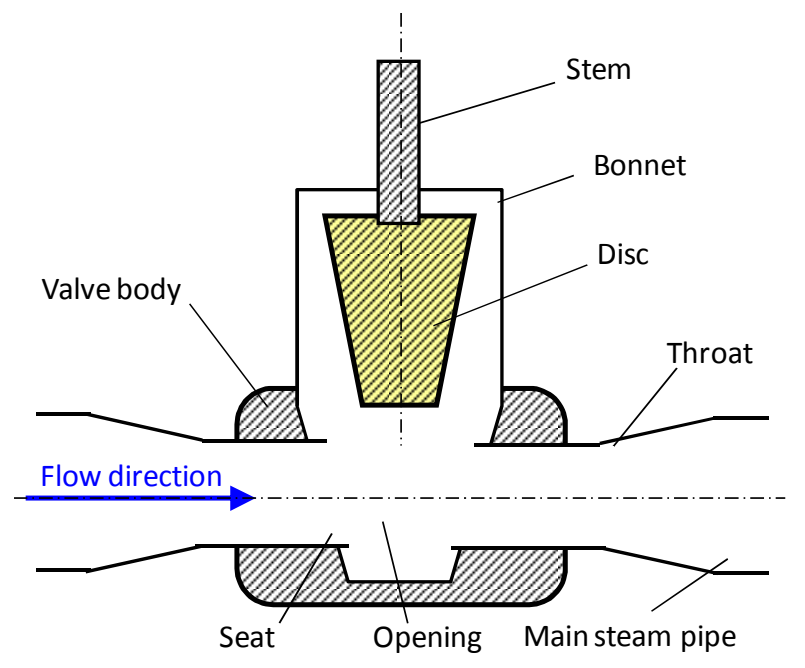

Fig 1. Schematic diagram of main steam stop valve. 


\section{Numerical Analysis Method}

In this section, we introduce the governing equations of the FDLBM $^{(13)-(22)}$ which is used for numerical analyses of the MSV. As we describe in the Introduction, the FDLBM is unlike conventional CFD methods, and is based on molecular gas dynamics. The FDLBM can be derived from the Boltzmann equation which is the basic equation for molecular gas dynamics. In the FDLBM, the density $\rho$ and the flow velocity $u_{\alpha}$ are given from the velocity distribution function $f_{i}$ taking the moments of the molecular velocities $c_{i \alpha}$ as

$$
\begin{aligned}
& \rho=\sum_{i=1}^{I} f_{i}, \\
& u_{\alpha}=\frac{1}{\rho} \sum_{i=1}^{I} f_{i} c_{i \alpha},
\end{aligned}
$$

where the respective subscripts $i$ and $\alpha$ indicate the molecular velocity direction and the spatial direction in the Cartesian coordinates and $I$ is the number of directions of the molecular velocities.

The equation to be solved in the FDLBM is the following kinetic equation (discretevelocity Bhatnagar-Gross-Krook (BGK) equation):

$$
\frac{\partial f_{i}}{\partial t}+c_{i \beta} \frac{\partial f_{i}}{\partial x_{\beta}}=\frac{f_{i}^{e q}-f_{i}}{\tau}-\frac{A}{\tau} c_{i \beta} \frac{\partial f_{i}^{e q}-f_{i}}{\partial x_{\beta}},
$$

where $t$ and $x_{\beta}$ are the time and the Cartesian coordinates, respectively. The first term of the right-hand side shows the lattice BGK collision operator. $f_{i}^{e q}$ is the local equilibrium distribution function which is calculated by macroscopic variables. The relaxation time $\tau$ represents the rate of approach to the equilibrium state. The last term of the right-hand side maintains numerical stability of the FDLBM when the molecular viscosity $\mu$ is set to a small value. ${ }^{(18)}$

In order to apply the FDLBM to the numerical analysis of flow-acoustic interactions in the MSV, improvements of its computational efficiency are necessary. In the previous study, we developed improvements for computational acceleration of the FDLBM. Most of the computations of the FDLBM are for calculations of the spatial-derivative terms and the equilibrium distribution function. Eq. (3) includes three spatial-derivative terms. Uniting these derivative terms leads to computational acceleration of the FDLBM. To do this, we proposed a new function $\phi_{i}$ instead of the equilibrium distribution function $f_{i}^{e q}$. The new function consists of the distribution function and the equilibrium distribution function ${ }^{(22)}$

$$
\phi_{i}=\left(1-\frac{A}{\tau}+\frac{B}{\tau}\right) f_{i}+\left(\frac{A}{\tau}-\frac{B}{\tau}\right) f_{i}^{e q}
$$

where the new function satisfies the following equations:

$$
\begin{aligned}
& \sum_{i=1}^{I} \phi_{i}=\rho, \\
& \sum_{i=1}^{I} \phi_{i} c_{i \alpha}=\rho u_{\alpha},
\end{aligned}
$$

and $A$ is a constant corresponding to the molecular (laminar) viscosity $\mu . B$ is the variable related to the turbulent viscosity $\mu_{T} . A$ and $B$ are defined by the following equations:

$$
\begin{aligned}
& A=\tau-\frac{\mu}{c^{2}}, \\
& B=\frac{\mu_{T}}{c^{2}},
\end{aligned}
$$

where $c$ represents the speed of sound. Despite the single relaxation time $\tau$, we can independently set the values of $\mu$ and $\mu_{T}$ by introducing $A$ and $B$. The governing equation of the FDLBM using the new function $\phi_{i}$ is

$$
\frac{\partial f_{i}}{\partial t}+c_{i \beta} \frac{\partial \phi_{i}}{\partial x_{\beta}}=\frac{\phi_{i}-f_{i}}{A-B} \text {. }
$$


Computational efficiency of the FDLBM can be increased by using Eq. (9) instead of Eq. (3) because the number of the spatial-derivative terms is reduced. Chapman-Enskog analysis shows that Eq. (9) is equivalent to Eq. (3). In this analysis, the distribution function $f_{i}$ and the function $\phi_{i}$ are expanded about the equilibrium distribution function $f_{i}^{e q}$ :

$$
\begin{aligned}
& f_{i}=f_{i}^{e q}+\varepsilon f_{i}^{(1)}+\varepsilon^{2} f_{i}^{(2)}+\cdots, \\
& \phi_{i}=f_{i}^{e q}+\left(1-\frac{A}{\tau}+\frac{B}{\tau}\right) \varepsilon f_{i}^{(1)}+\left(1-\frac{A}{\tau}+\frac{B}{\tau}\right) \varepsilon^{2} f_{i}^{(2)}+\cdots,
\end{aligned}
$$

where $\varepsilon$ is a small parameter. Substituting Eqs. (10) and (11) into Eq. (9), we obtain the following equation.

$$
\frac{\partial}{\partial t}\left(f_{i}^{e q}+\varepsilon f_{i}^{(1)}\right)+c_{i \beta} \frac{\partial}{\partial x_{\beta}}\left(f_{i}^{e q}+\left(1-\frac{A}{\tau}+\frac{B}{\tau}\right) \varepsilon f_{i}^{(1)}\right)=-\frac{1}{\tau} \varepsilon f_{i}^{(1)}+O\left(\varepsilon^{2}\right),
$$

With some algebraic calculations, the Navier-Stokes equations are derived from Eq. (12). As the molecular velocity model, the 3-dimensional 13-velocity model (D3Q13) ${ }^{(21)}$ whose computational efficiency is superior to that of D3Q15 is employed. The molecular velocities $c_{i \alpha}$ of D3Q13 are shown in Fig. 2 and Table 1. The speed of sound $c$ and the pressure $p$ are obtained by $c=c_{0} \sqrt{2 / 15}$ and $p=\rho c^{2}$, respectively. Isothermal compressible fluid in which the speed of sound is constant is derived from D3Q13. In low Mach number flows, the error of the isothermal assumption becomes $\mathrm{O}\left(\mathrm{Ma}^{2}\right)$. The error is negligible in our simulations since the Mach number is less than 0.2. In this model, the function $\phi_{i}$ is shown as

$$
\begin{array}{r}
\phi_{1}=\left(1-\frac{A}{\tau}+\frac{B}{\tau}\right) f_{1}+\left(\frac{A}{\tau}-\frac{B}{\tau}\right) \rho\left(\frac{3}{5}-\frac{2}{15} \frac{u_{\beta}^{2}}{c^{2}}\right) \\
\phi_{i}=\left(1-\frac{A}{\tau}+\frac{B}{\tau}\right) f_{i}+\left(\frac{A}{\tau}-\frac{B}{\tau}\right) \rho\left(\frac{1}{30}-\frac{1}{60} \frac{u_{\beta}^{2}}{c^{2}}+\frac{1}{30} \frac{u_{\beta} c_{i \beta}}{c^{2}}+\frac{1}{90} \frac{\left(u_{\beta} c_{i \beta}\right)^{2}}{c^{4}}\right) . \\
i=2,3, \cdots, 13
\end{array}
$$

The 4-stage second-order Runge-Kutta method is used for time integration of Eq. (9) and that method is as follows.

$$
\left\{\begin{array}{l}
f_{i}^{(1)}=f_{i}^{n}-\frac{1}{3} \Delta t\left(c_{i \alpha} \frac{\partial \phi_{i}^{n}}{\partial x_{\alpha}}+\frac{f_{i}^{n}-\phi_{i}^{n}}{A-B^{n}}\right) \\
f_{i}^{(2)}=f_{i}^{n}-\frac{4}{15} \Delta t\left(c_{i \alpha} \frac{\partial \phi_{i}^{(1)}}{\partial x_{\alpha}}+\frac{f_{i}^{(1)}-\phi_{i}^{(1)}}{A-B^{(1)}}\right) \\
f_{i}^{(3)}=f_{i}^{n}-\frac{5}{9} \Delta t\left(c_{i \alpha} \frac{\partial \phi_{i}^{(2)}}{\partial x_{\alpha}}+\frac{f_{i}^{(2)}-\phi_{i}^{(2)}}{A-B^{(2)}}\right) \\
f_{i}^{n+1}=f_{i}^{n}-\Delta t\left(c_{i \alpha} \frac{\partial \phi_{i}^{(3)}}{\partial x_{\alpha}}+\frac{f_{i}^{(3)}-\phi_{i}^{(3)}}{A-B^{(3)}}\right)
\end{array}\right.
$$

In order to increase simulation accuracy, the spatial derivative of Eq. (9) is discretized by the sixth-order central-difference scheme as follows.

$$
\begin{array}{r}
c_{i \alpha} \frac{\partial \phi_{i}}{\partial x_{\alpha}}=c_{i \alpha} \frac{-\phi_{i}\left(x_{\alpha}-3 \Delta x_{\alpha}\right)+9 \phi_{i}\left(x_{\alpha}-2 \Delta x_{\alpha}\right)-45 \phi_{i}\left(x_{\alpha}-\Delta x_{\alpha}\right)}{60 \Delta x_{\alpha}} \\
+\frac{45 \phi_{i}\left(x_{\alpha}+\Delta x_{\alpha}\right)-9 \phi_{i}\left(x_{\alpha}+2 \Delta x_{\alpha}\right)+\phi_{i}\left(x_{\alpha}+3 \Delta x_{\alpha}\right)}{60 \Delta x_{\alpha}}
\end{array}
$$

The turbulent viscosity is calculated by the lattice Boltzmann subgrid model. ${ }^{(29)}$ As the lattice Boltzmann subgrid model, we employ the Smagorinsky eddy viscosity model, which computes the turbulent viscosity $\mu_{T}$ from the local shear rate and the filter size:

$$
\mu_{T}=C \Delta^{2} \rho \sqrt{2 S_{\alpha \beta} S_{\alpha \beta}},
$$

where $S_{\alpha \beta}$ is the resolved strain rate tensor and $\sqrt{2 S_{\alpha \beta} S_{\alpha \beta}}$ is the local intensity of the strain tensor. The velocity distribution function is filtered by Favre averaging. The filter size $\Delta$ is computed as the cubic root of the grid volume. The Smagorinsky coefficient $C$ is 
computed by the dynamic Smagorinsky model. ${ }^{(30)}$

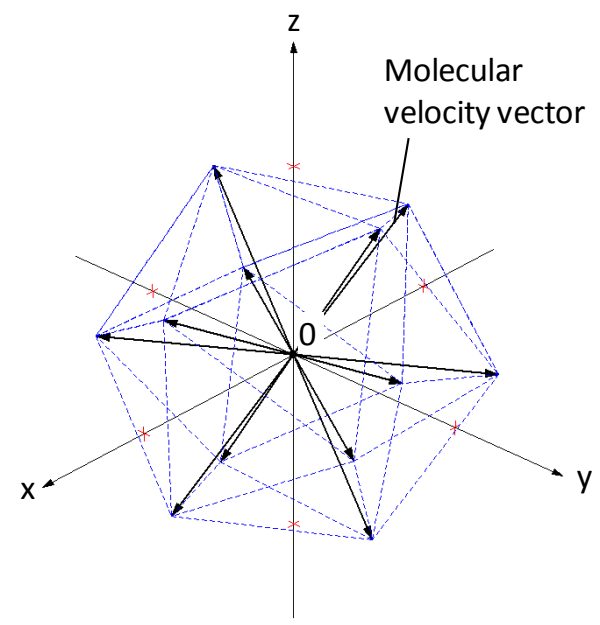

Fig 2. Distribution of the molecule velocities $c_{i \alpha}$.

Table 1 Cartesian components of the molecule velocities, where $c_{1}=c_{0} \sqrt{10 /(5+\sqrt{5})}$ and $c_{2}=c_{0}(1+\sqrt{5}) \sqrt{5 / 2(5+\sqrt{5})}$.

\begin{tabular}{||c|c|c|c||}
\hline$I$ & $C_{I X}$ & $C_{I Y}$ & $C_{I Z}$ \\
\hline 1 & 0 & 0 & 0 \\
\hline 2 & 0 & $c_{1}$ & $c_{2}$ \\
\hline 3 & 0 & $-c_{1}$ & $c_{2}$ \\
\hline 4 & 0 & $c_{1}$ & $-c_{2}$ \\
\hline 5 & 0 & $-c_{1}$ & $-c_{2}$ \\
\hline 6 & $c_{2}$ & 0 & $c_{1}$ \\
\hline 7 & $-c_{2}$ & 0 & $c_{1}$ \\
\hline 8 & $c_{2}$ & 0 & $-c_{1}$ \\
\hline 9 & $-c_{2}$ & 0 & $-c_{1}$ \\
\hline 10 & $c_{1}$ & $c_{2}$ & 0 \\
\hline 11 & $-c_{1}$ & $c_{2}$ & 0 \\
\hline 12 & $c_{1}$ & $-c_{2}$ & 0 \\
\hline 13 & $-c_{1}$ & $-c_{2}$ & 0 \\
\hline
\end{tabular}

\section{Flow-Acoustic Interaction Simulations}

\subsection{Experimental Apparatus}

We applied the FDLBM to simulations of flow-acoustic interactions in the MSV. Our analysis object was the MSV model of the 1/5-scale test apparatus illustrated in Fig. 3. This test apparatus is composed of an air compressor, a flow regulating valve and an acrylic MSV model. Air at ordinary temperature and almost atmospheric pressure is used as fluid instead of steam flow. Physical properties of the fluid are shown in Table 2. In order to suppress the influence of external pressure fluctuations due to the air compressor, silencers are installed upstream and downstream from the MSV model. The flow rate is adjusted by the flow regulating valve.

A photo of the MSV model with the gate valve is shown in Fig. 4. The MSV model is composed of the valve body, throats (one before and one after the MSV model) and the bonnet. It is known that a strong sound occurs in the MSV when the disk is removed. Thus, 
the MSV model targets the MSV without the disk. Pressure fluctuations are measured by the pressure sensor installed on top of the bonnet.

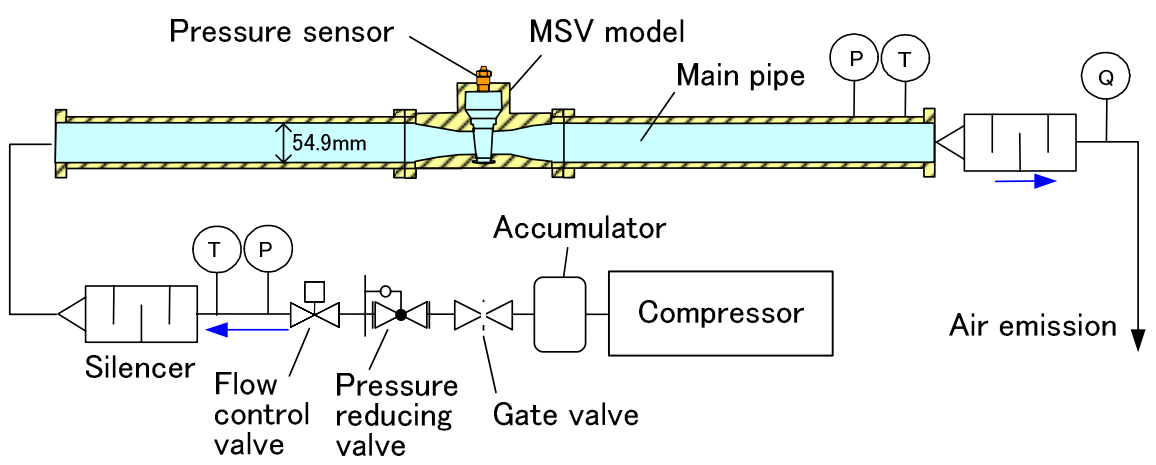

Fig. 3 Schematic presentation of the test facility using air at ambient conditions.

Table 2 Physical properties of the fluid.

\begin{tabular}{||l|l||}
\hline ITEM & VALUE \\
\hline \hline Temperature & $20[\mathrm{deg} \mathrm{C}]$ \\
\hline Density & $1.204\left[\mathrm{~kg} / \mathrm{m}^{3}\right]$ \\
\hline Pressure & $101.3[\mathrm{kPa}]$ \\
\hline Speed of sound & $343.7[\mathrm{~m} / \mathrm{s}]$ \\
\hline Molecular viscosity & $1.808 \times 10^{-5}[\mathrm{~Pa} \mathrm{~s}]$ \\
\hline
\end{tabular}

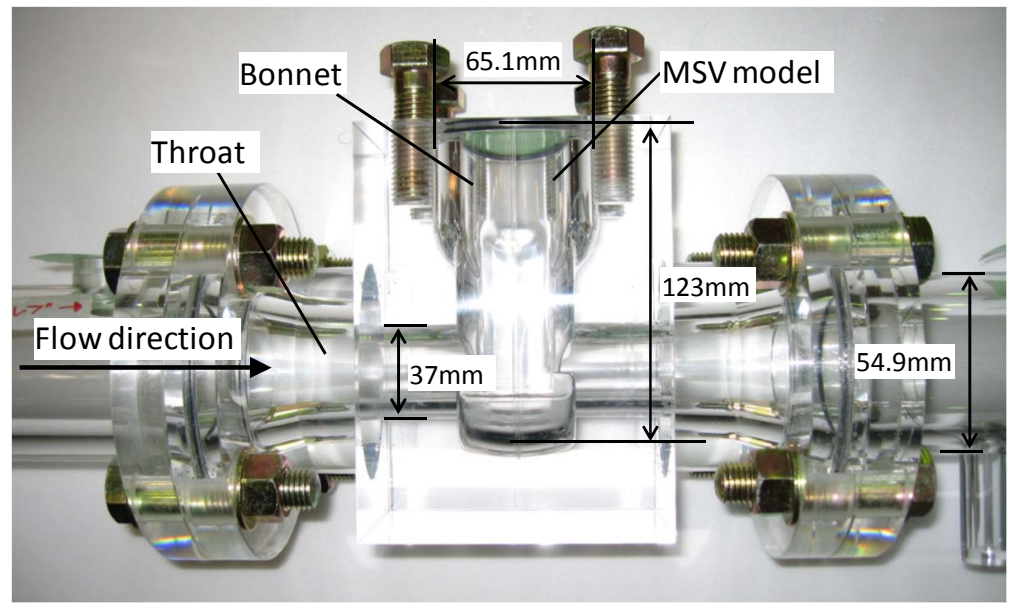

Fig. 4 Photo of the main steam stop valve model.

\subsection{Analysis Conditions}

The multi-block structure grid which is shown in Fig. 5 was used for three-dimensional simulations of flow-acoustic interaction (hole-tone) in the MSV model. The total number of grid points is about $1,000,000$. The minimum grid width $\Delta x$ is $0.3 \mathrm{~mm}$. The CFL number $c \Delta t / \Delta x$ is 1.0 , where $\Delta t$ is the time increment. On the wall boundary, the flow velocity 
is given by the wall function defined by the Spaulding law and the density is given by the Neumann type boundary condition. On the inlet boundary, the velocity is constant and the Neumann type boundary condition is applied for the density. On the outlet boundary, the density is constant and the Neumann type boundary condition is applied for the velocity. In order to prevent acoustic waves generated in the MSV model from reflecting at the inlet or the outlet boundaries, sponge regions which attenuate the acoustic waves by numerical viscosity are placed as shown in Fig. 5.

The non-dimensional parameters of this problem are the Mach number $M a$ and the Reynolds number $R e$. The Mach number $M a$ is defined by the following equation:

$$
M a=\frac{U}{c}
$$

where $U$ is the mean flow velocity at the main pipe. The Reynolds number $R e$ is defined by the following equation:

$$
R e=\frac{U d}{v}
$$

where $d$ is the inner diameter of the main pipe and $v$ is the kinematic viscosity. The Mach number $M a$ is changed from 0.07 to 0.12 . The Reynolds number $R e$ is also changed from 88,000 to 150,000 .
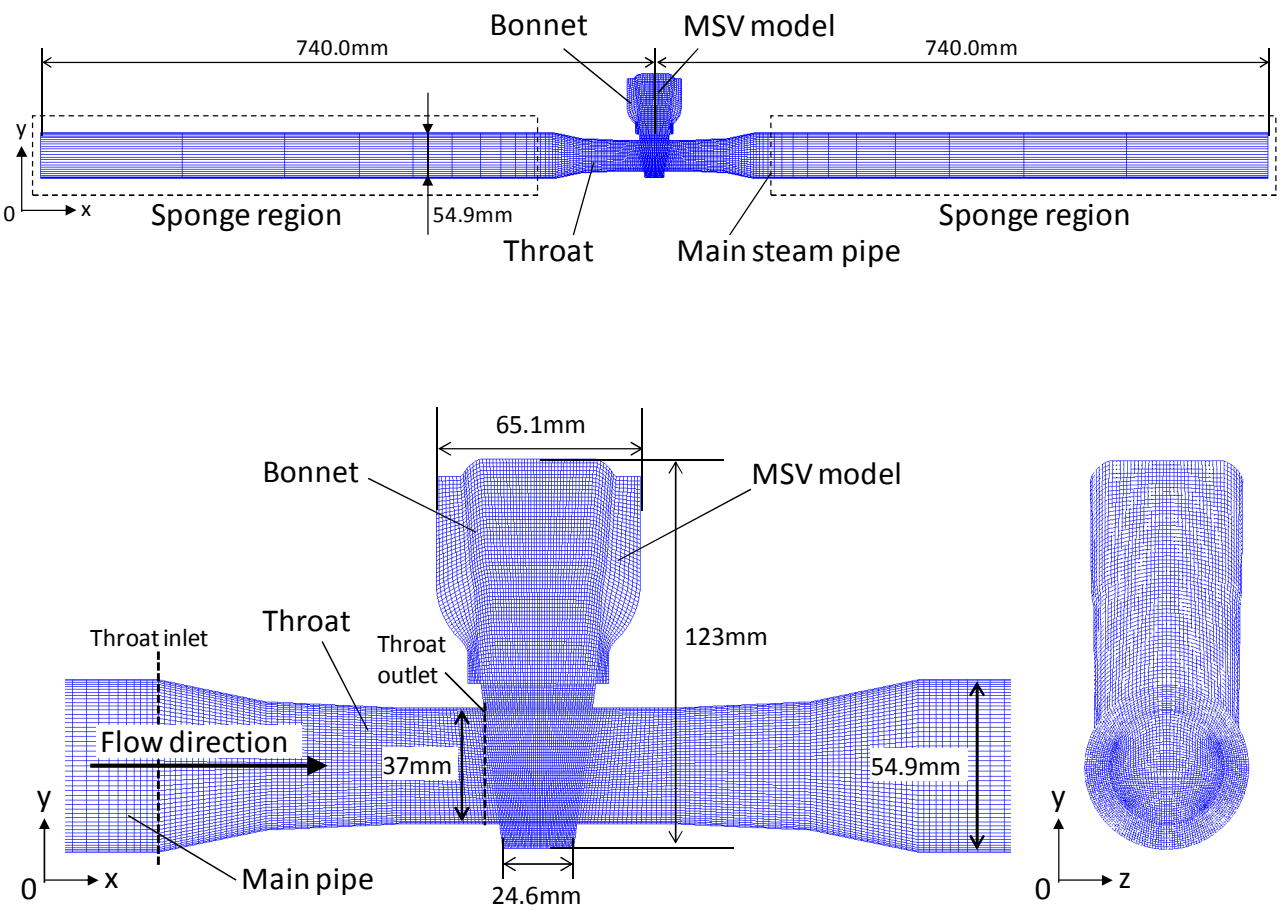

Fig. 5 Computational mesh for simulations of the flow-acoustic interaction (hole-tone)

\subsection{Analysis Results}

Time-averaged flow velocity $\bar{u}$ in the throat inlet and outlet are shown in Fig. 6. Flow velocity profile at the inlet of the throat becomes that of fully-developed turbulence. At the outlet of the throat, the flow is accelerated due to reduction of cross-sectional area.

For validation of the analysis results, the pressure fluctuations on the top of the bonnet are compared with those of the experiment. Fig. 7 compares the pressure fluctuations 
at $M a=0.07$. Both sets of results show random waveforms with small amplitudes. It is likely that the self-sustained sound has not occurred since the acoustic pressure of the pressure fluctuations is small compared with the dynamic pressure. Fig. 8 compares the pressure fluctuations at $M a=0.12$. Both sets of results show substantial sinusoidal waveforms. The acoustic pressure becomes larger compared with that of $M a=0.07$. The pressure spectra of these pressure fluctuations are shown in Fig. 9. The numerical results show good agreement with the experimental data, with respect to the spectral peak around $2,900 \mathrm{~Hz}$. The spectral peak at $3,800 \mathrm{~Hz}$ is observed in the numerical result. It is likely that the reflection of the acoustic waves in the bonnet by numerical errors is the cause. However, affect of these errors on the flow is small because the peak value is negligible compared with that of the spectral peak around $2,900 \mathrm{~Hz}$.

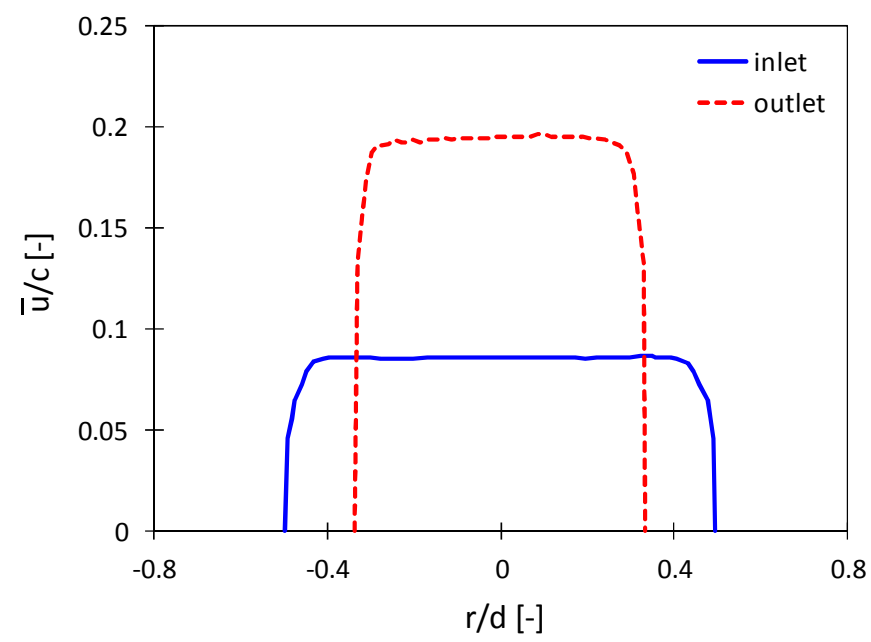

Fig. 6 Time-averaged flow velocity $\bar{u}$ profiles in the throat at $M a=0.07$.

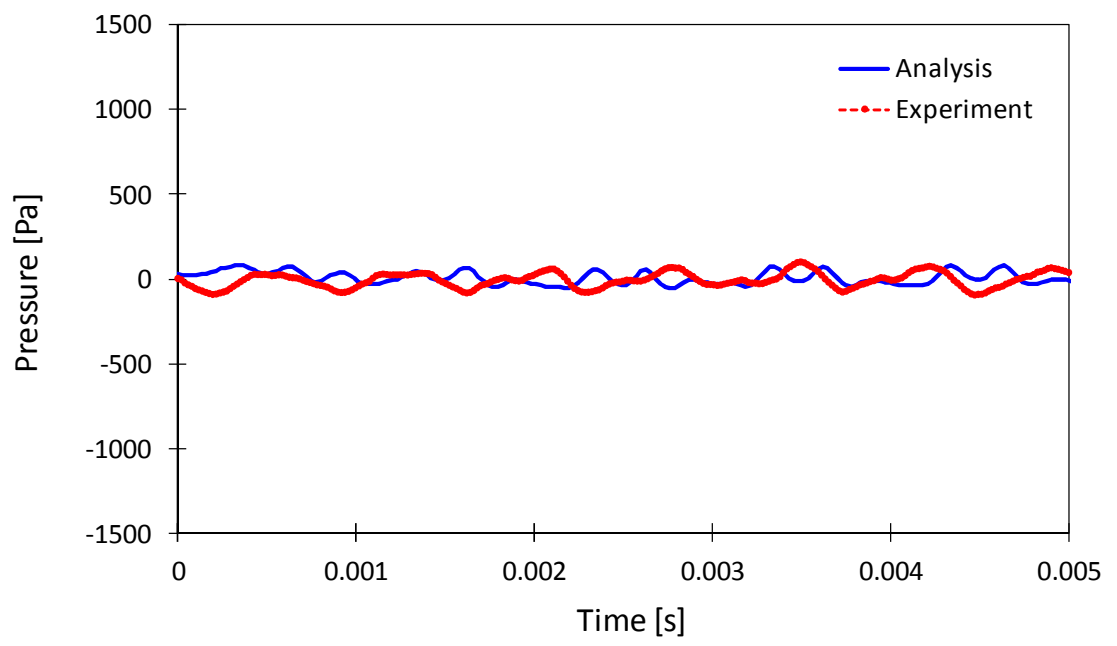

Fig. 7 Pressure fluctuations on the top of the bonnet at $M a=0.07$. 


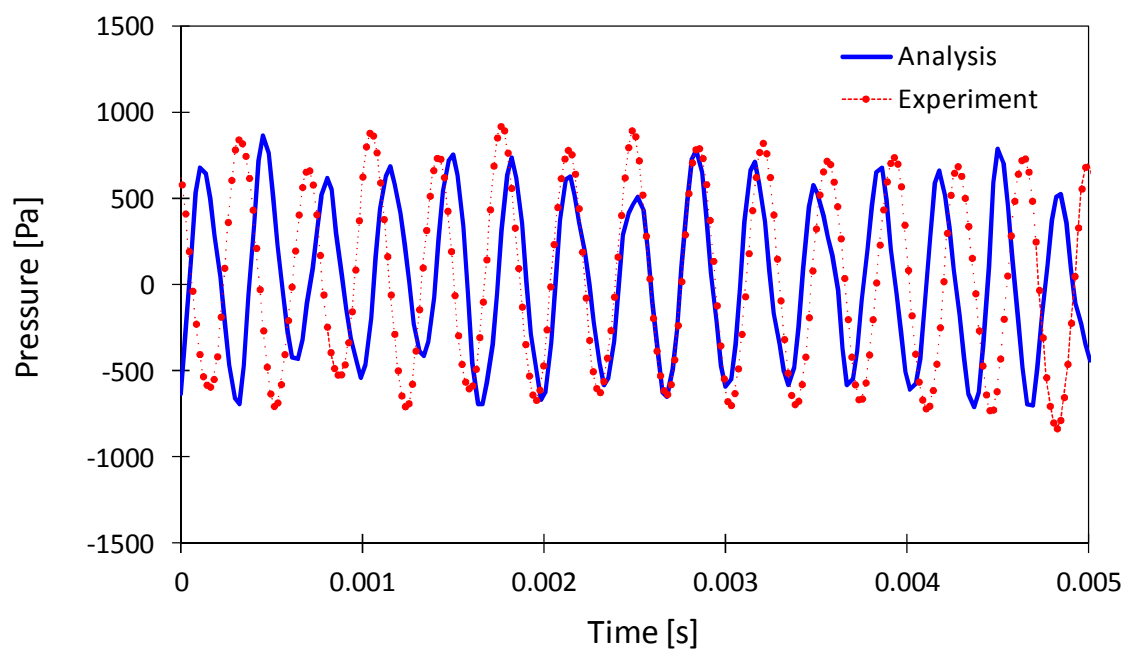

Fig. 8 Pressure fluctuations on the top of the bonnet at $M a=0.12$.

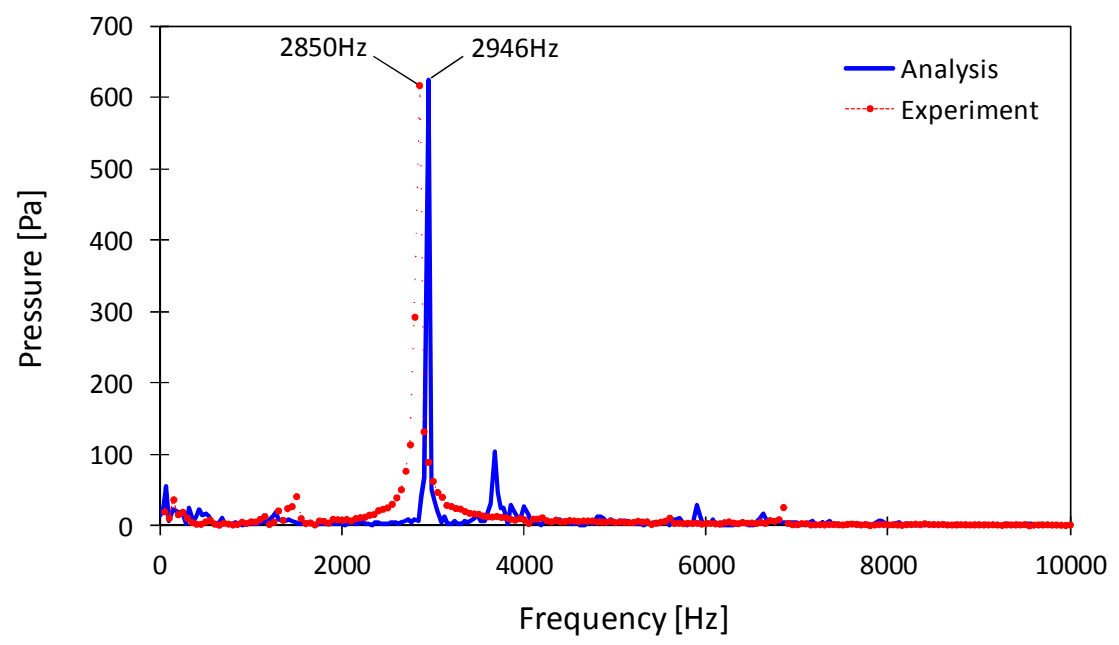

Fig. 9 Comparison of pressure spectra on the top of the bonnet at $M a=0.12$.

In order to investigate the mechanism of the sound in the MSV, we show the vorticity field of the simulation results in Fig. 10, where $T$ represents the cycle of the unsteady vortex motion. The vorticity $\omega$ is non-dimensionalized by the inner diameter of the main pipe $d$ and the speed of sound $c$. Anti-clockwise vorticity is defined as positive. The Mach number is 0.12 which corresponds to the flow condition where the large pressure fluctuations occur. There are two vortex rings in the opening (Fig. 10). The vortex ring is periodically generated at the upstream edge (Fig. 10(a)). The vortex ring has a slope along the upstream edge. The vortex ring grows and is convected downward while reducing its slope (Figs. 10 (b) and (c)). Finally, the vortex ring collides with the downstream edge (Fig. 10 (d)). The vortex ring begins to collide with the bottom of the edge since the downstream edge has a slope. The pressure field of the simulation results is shown in Fig. 11. The pressure $p$ is non-dimensionalized by $c^{2}$ and the density $\rho$. The pressure fluctuations are periodically generated around the downstream edge. It is likely that the collision of the vortex rings causes generation of the pressure fluctuations. These results indicate that the hole-tone is generated in the MSV. The pressure field including the bonnet is shown in Fig. 12. Acoustic resonance in the vertical direction occurs in the MSV. A standing wave having nodes at the center of the bonnet and the opening is generated by the acoustic resonance. 
Frequency of this standing wave is about $2,800 \mathrm{~Hz}$, which agrees closely with the frequency of the peak value in Fig. 9. From these results, we clarify that the sound of the MSV is generated by the hole-tone in the opening and the acoustic resonance in the vertical direction of the MSV.

\section{Suppression Method of Sound in MSV}

\subsection{Tabs for Suppression of Sound}

In this section, we propose a suppression method of the sound generated in the MSV. From the numerical analyses, we clarified that the sound of the MSV is generated by the hole-tone in the opening. Nakazono proposed the suppression method of the hole-tone, where triangular tabs are installed at the inlet nozzle. They indicated that the height of the triangular tabs needs to be at least $10 \%$ of the inner diameter in the nozzle. In order to study
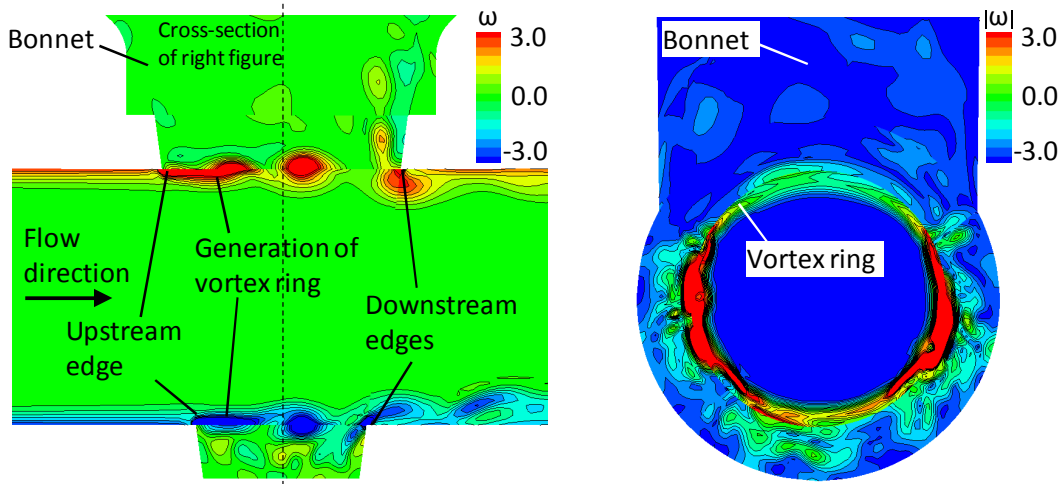

(a) $t=0$
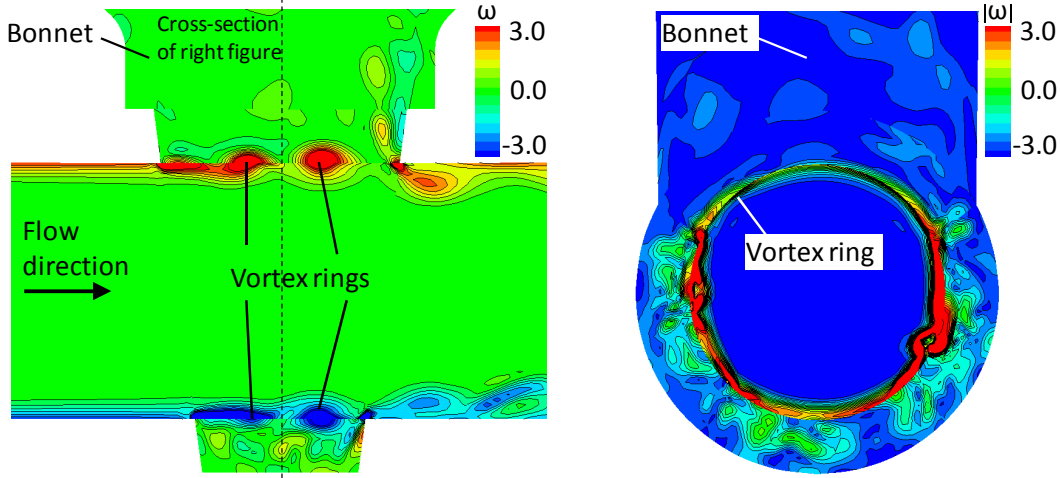

(b) $t=T / 4$
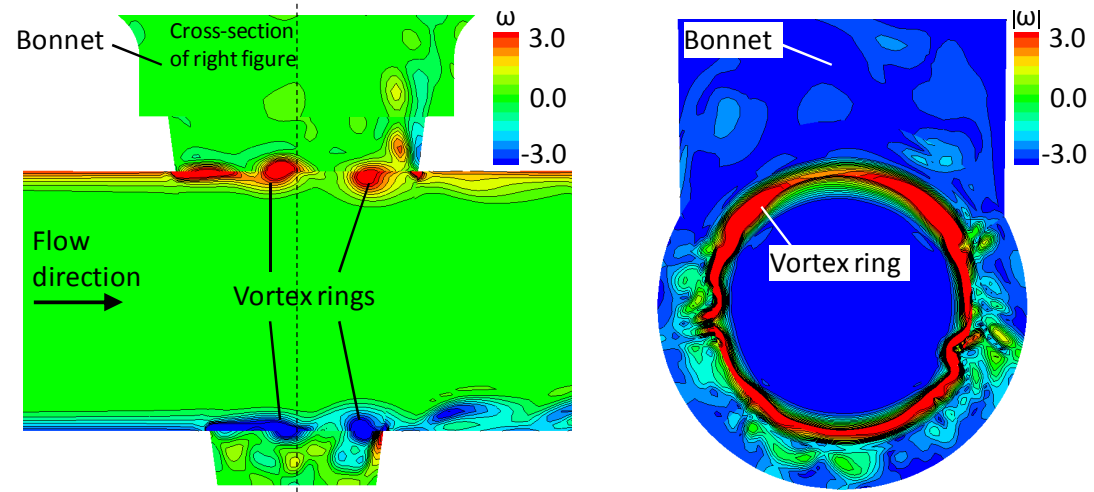

(c) $t=T / 2$ 

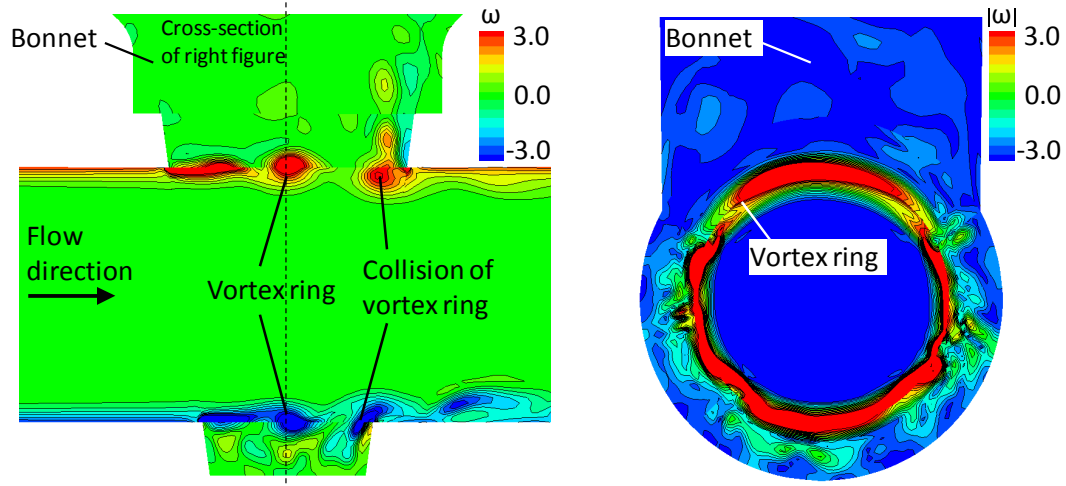

(d) $t=3 T / 4$

Fig. 10 Behaviors of the vortex rings in the main steam stop valve (left, vorticity of z-direction; right, amplitude of vorticity).

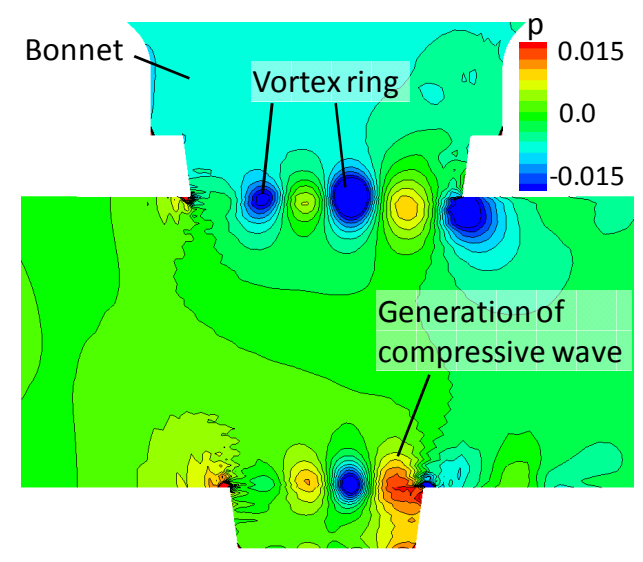

(a) $t=0$

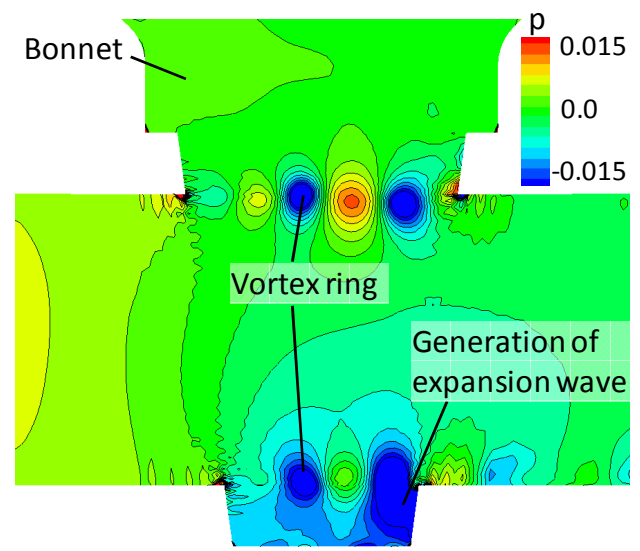

(c) $t=T / 2$

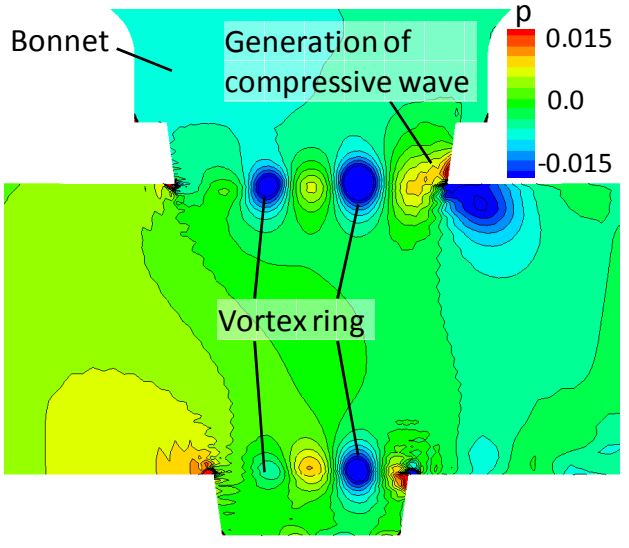

(b) $t=T / 4$

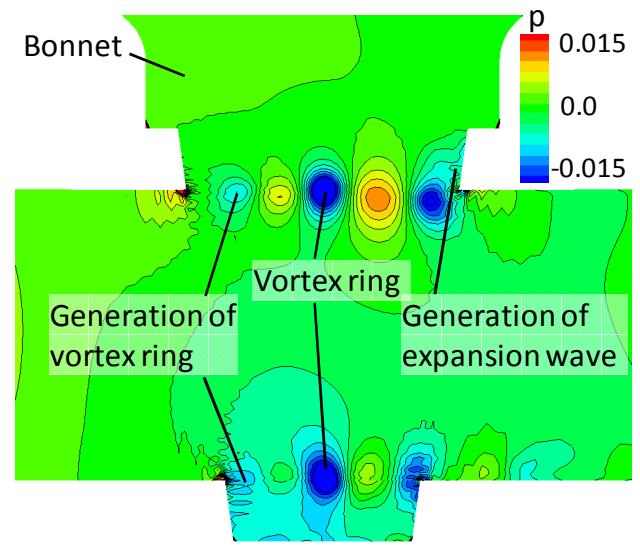

(d) $t=3 T / 4$

Fig. 11 Generation of acoustic waves around the downstream edges due to the vortex rings. 


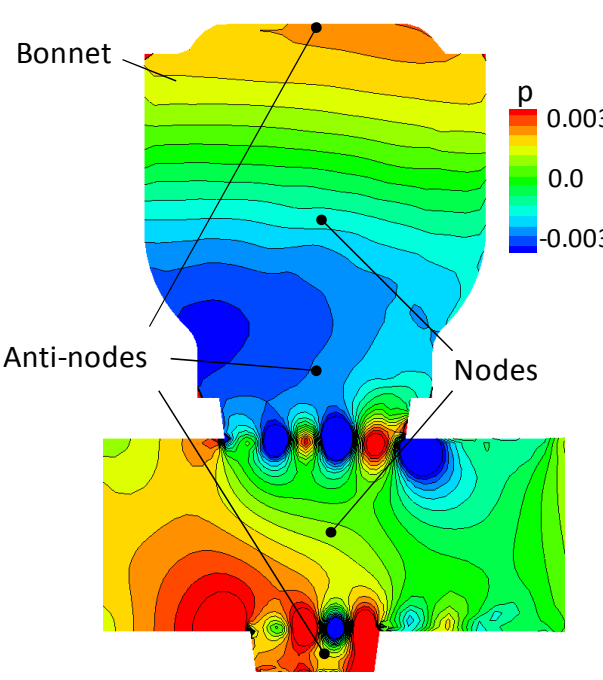

(a) $t=0$

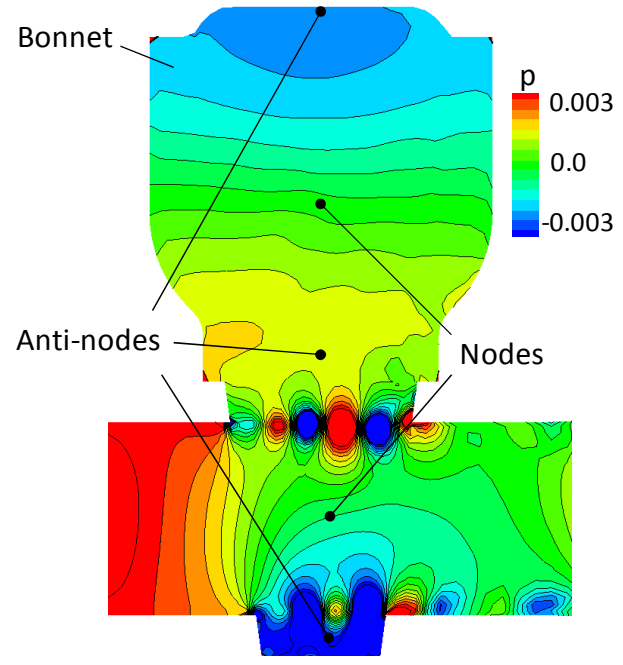

(b) $t=\mathrm{T} / 2$

Fig. 12 Acoustic resonance in the main steam stop valve model

effects of the triangle tabs on the suppression of the sound in the MSV, we made the MSV model with tabs for an experiment. This model is shown in Fig. 13. Its shape is slightly different from that of the previous model shown in Fig. 4. The bonnet volume of this model is small compared with that of the previous model. Eight triangular tabs having a height of $15 \%$ of the inner diameter in the nozzle are installed at the inlet seat. This number of tabs was recommended by Nakazono. In this study, we also tested slanted tabs. As shown in Fig. 14(b), the slanted tabs are placed at an angle of 45 degrees to the flow. The height of the slanted tabs is $7.5 \%$ of the inner diameter in the nozzle. We expected that the longitudinal vortex generated by the slanted tab would prevent the vortex ring from forming in the MSV. The test apparatus shown in Fig. 3 is used for the experiment on the MSV with the tabs.

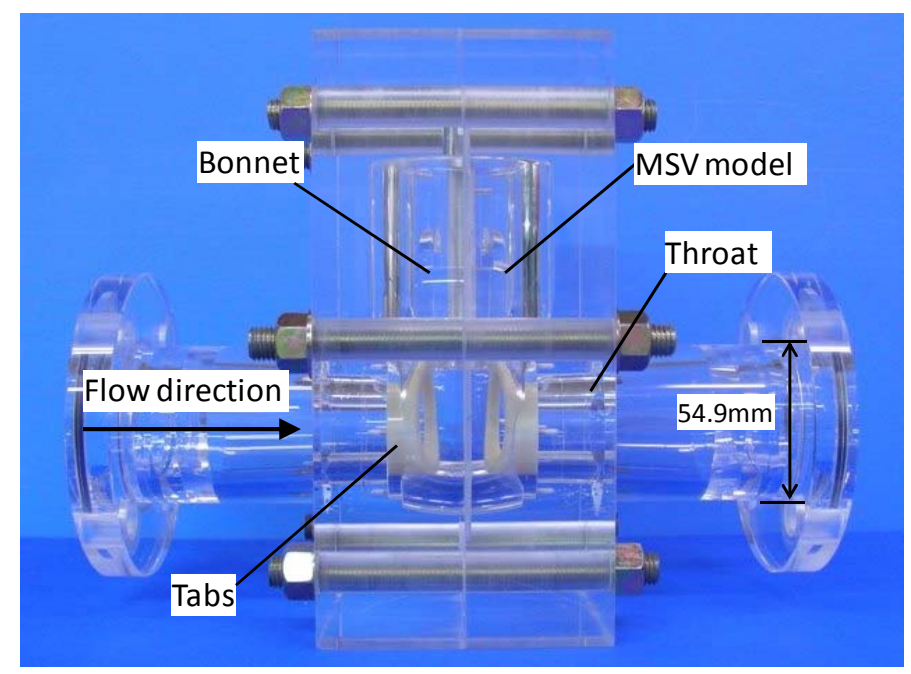

Fig. 13 Appearance of the MSV model with tabs. 


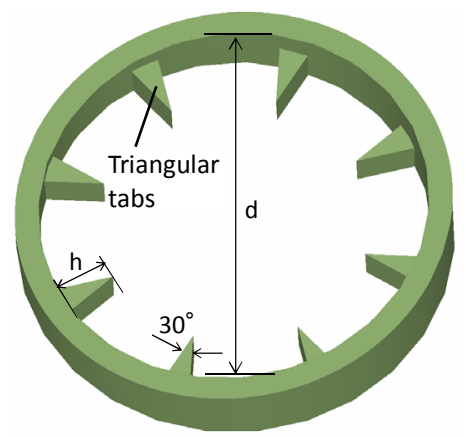

(a) Triangular tabs

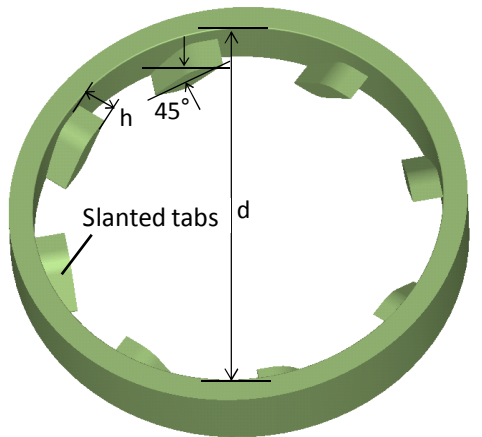

(b) Slanted tabs

Fig. 14 Schematic of the tabs used for suppression of sound in the MSV.

\subsection{Experimental Results}

Figures 15 and 16 compare the pressure spectra on the top of the bonnet. The Mach number is 0.18 , which corresponds to the conditions under which the powerful sound was generated. The Mach number at the resonant condition differs from the previous MSV model by difference of the bonnet volume. The pressure and the frequency are normalized by the peak value for conditions of no tabs. In the MSV with the triangular tabs, the peak value of the pressure decreases by $60 \%$ compared with the MSV without tabs. However, the sound by the hole-tone is not suppressed completely. We think that the triangular tabs cannot prevent the vortex rings from forming in this MSV. The shape of the triangular tabs is symmetric in the circumferential direction. Thus, the circumferential flow induced by the tabs is small, and the vortex rings remain in the MSV. In the MSV with the slanted tabs, the peak value of the pressure vanishes although its height is low compared with the triangular tabs. The slanted tabs have an angle with respect to the main flow. The pressure difference occurs between the upstream and downstream surfaces of the slanted tabs. The pressure difference and the angle with respect to the main flow cause the circumferential flow or the longitudinal vortex by a mechanism similar to a vortex generator. These circumferential disturbances prevent the vortex rings from forming in the MSV. These results show that the slanted tabs are effective in suppression of the hole-tone compared with the triangular tabs. However, it should be noted that the cross-section of the slanted tabs is larger than that of the triangular tabs. Thus, the slanted tabs might have a high pressure loss compared with the triangular tabs. On this point, further experiments and analyses are needed. We checked the effect of these tabs within the conditions of this study; further research is required to determine an extended application range of these tabs. 


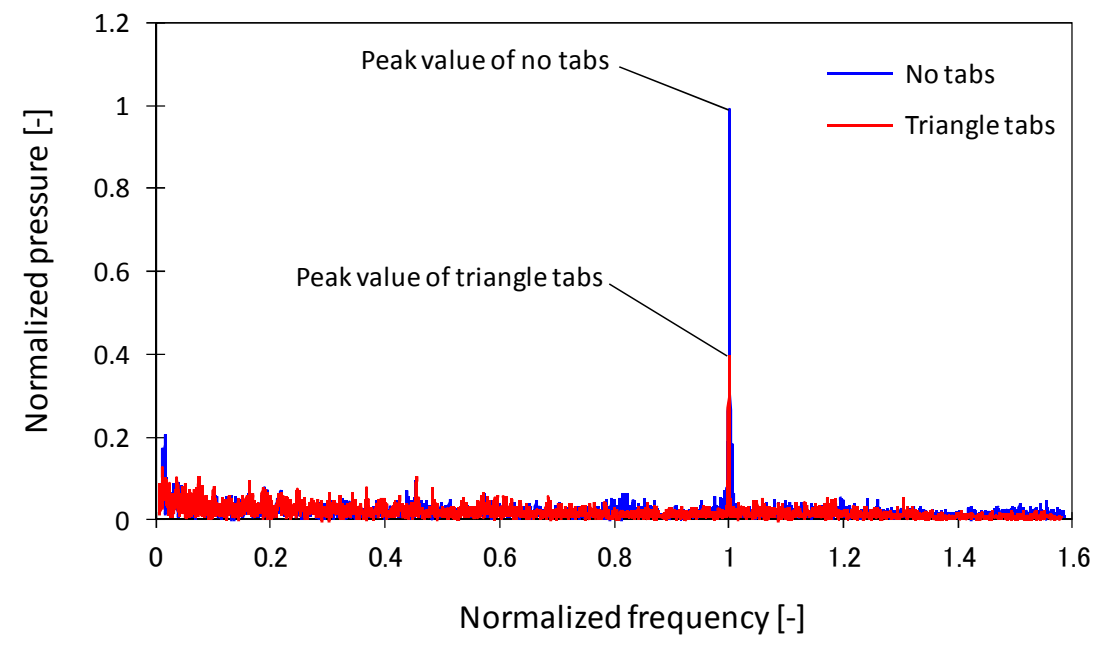

Fig. 15 Comparison of pressure spectra on the top of the bonnet.

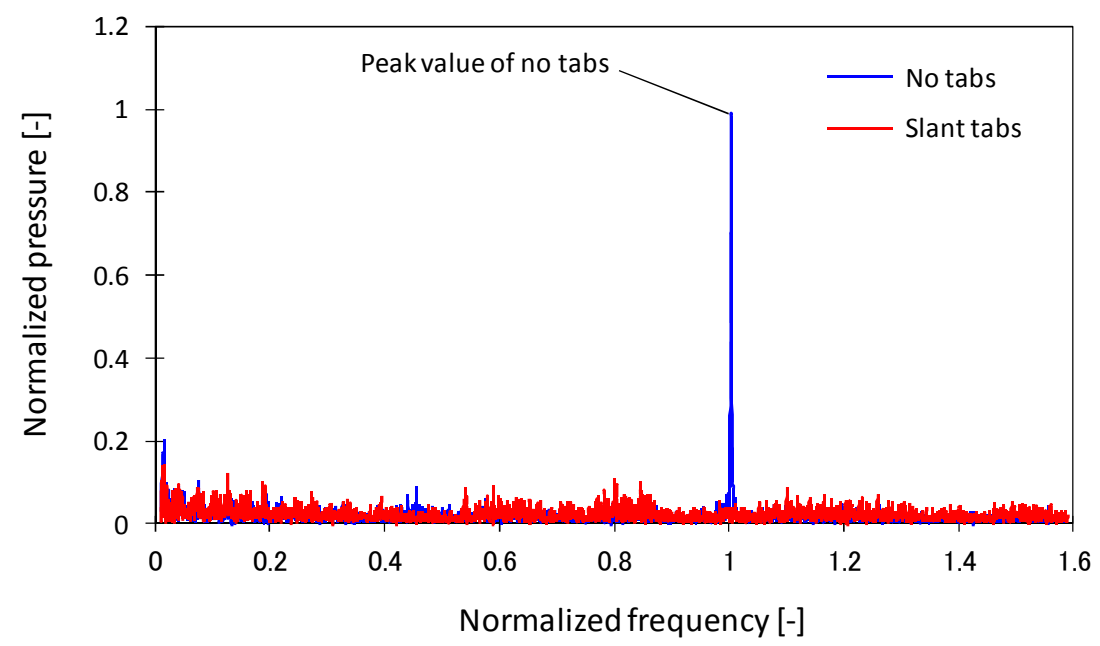

Fig. 16 Comparison of pressure spectra on the top of the bonnet.

\section{Conclusions}

In this article, we investigated the mechanism of the sound suppression in the MSV using the numerical analyses. As the numerical analysis method, the FDLBM which had been computationally accelerated was applied. The numerical results were validated by the experiment in which the $1 / 5$-scale model of a typical MSV consisting of the gate valve was used. We also investigated the suppression method of the sound generation in the MSV. Effects of the proposed suppression method were tested experimentally. The following results were obtained.

(1) For validation of the analysis results, the pressure fluctuations on the top of the bonnet were compared with those of the experiment. Both sets of results showed substantial sinusoidal waveforms at $M a=0.12$. The analysis results had good agreement with the experimental data. Vortex rings were periodically generated at the upstream edge. Acoustic waves were periodically generated in the MSV by interaction between these vortex rings and the downstream edge. From the analysis results, it was clarified that the sound of the 
MSV was generated by the hole-tone in the opening and acoustic resonance in the vertical direction of the MSV.

(2) In order to prevent formation of the vortex rings in the opening which leads to the hole-tone, triangular or slanted tabs were installed at the inlet seat. Eight triangular tabs having a height of $15 \%$ of the inner diameter in the seat could reduce the peak value of the sound pressure by $60 \%$. Eight slanted tabs having the height of $7.5 \%$ of the inner diameter in the seat could suppress generation of the hole-tone in the MSV. These results showed that the slanted tabs are effective in suppression of the hole-tone compared with the triangular tabs. Further experiments and analyses with respect to the pressure loss are needed. The effect of these tabs was checked within the conditions of this study; further research is required to determine an extended application range of these tabs.

\section{References}

(1) Rayleigh, J. W. S., Theory of Sound, Vol. 2 (1945), pp. 410-412, Dover Publications.

(2) Rossiter, J. E., The Effect of Cavities on the Buffeting of Aircraft, Royal Aircraft Establishment Technical Memorandum, No. 754 (1962).

(3) Chanaud, R. C. and Powell, A., Some Experiments Concerning the Hole and Ring Tone, Journal of the Acoustic Society of America, Vol. 37, No. 5 (1965), pp.902-911.

(4) Rockwell, D. and Naudascher, E., Self-Sustained Oscillations of Impinging Free Shear Layers, Annual Review of Fluid Mechanics, Vol. 11, (1979), pp.67-94.

(5) Langthjem, M. A. and Nakano, M., A Numerical Simulation of the Hole-Tone Feedback Cycle Based on an Axisymmetric Discrete Vortex Method and Curl's Equation, Journal of Sound and Vibration, Vol. 288, No. 1-2, (2005), pp.133-176.

(6) Matsuura, K. and Nakano, M., Direct Computation of a Hole-Tone Feedback System at Very Low Mach Numbers, Journal of Fluid Science and Technology, Vol. 6, No. 4, (2011), pp. 548-561.

(7) Nonomura, T. and Fujii, K., Effects of difference scheme type in high-order weighted compact nonlinear schemes, Journal of Computational Physics, Vol. 228 No. 10, (2009), pp. 3533-3539.

(8) Nonomura, T., Iizuka, N. and Fujii, K., Freestream and vortex preservation properties of high-order WENO and WCNS on curvilinear grids, Computers and Fluids, Vol. 39, No. 2, (2010), pp. 197-214.

(9) Kawai, S. and Fujii, K., Compact scheme with filtering for large-eddy simulation of transitional boundary layer, AIAA Journal, Vol. 46, No. 3, (2008), pp.690-700.

(10) Yokoyama, H., Tsukamoto, Y., Kato, C. and Iida, A., Self-sustained oscillations with acoustic feedback in flow over a backward-facing step with a small upstream step, Physics of Fluids, Vol. 19, (2007), 106104.

(11) Yokoyama, H. and Kato, C., Fluid-acoustic interactions in self-sustained oscillations in turbulent cavity flows. I: Fluid-dynamic oscillations, Physics of Fluids, Vol. 21, (2009), 105103.

(12) Bogey, C. and Bailly, C., A family of low dispersive and low dissipative explicit schemes for flow and noise computations, Journal of Computational Physics, Vol. 194, No. 1, (2004), pp. 194-214.

(13) Nannelli, F. and Succi, S., The lattice Boltzmann equation on irregular lattices, Journal of Statistical Physics, Vol. 68, No. 3, (1992), pp. 401-407.

(14) Reider, M. B. and Sterling, J. D., Accuracy of discrete-velocity BGK models for the simulation of the incompressible Navier-Stokes equations, Computers and Fluids, Vol. 24, (1995), pp. 459-467.

(15) Cao, N., Chen, S., Jin, S. and Martinez, D., Physical symmetry and lattice symmetry in the lattice Boltzmann method, Physical Review E, Vol. 55, (1997), pp.21-24. 
(16) Watari, M. and Tsutahara, M., Two-dimensional thermal model of the finite difference lattice Boltzmann method with high spatial isotropy, Physical Review E, Vol. 67, (2003), 036306.

(17) Seta, T. and Takahashi, R., Numerical stability analysis of FDLBM, Journal of Statistical Physics, Vol. 107, (2002), pp. 557-572.

(18) Tsutahara, M., Kataoka, T., Shikata, K. and Takada, N., New model and scheme for compressible fluids of the finite difference lattice Boltzmann method and direct simulations of aerodynamic sound, Computers and Fluids, Vol. 37, (2008), pp. 79-89.

(19) Tamura, A. and Tsutahara, M., Direct simulation of Aeolian tones emitted from a circular cylinder in transonic flows using the finite difference lattice Boltzmann method, Fluid Dynamics Research, Vol. 42, (2010), 015007.

(20) Tamura, A., Tsutahara, M., Kataoka, T., Aoyama, T. and Yang, C., Numerical simulation of two-dimensional blade-vortex interactions using finite difference lattice Boltzmann method, AIAA Journal, Vol. 46 No. 9, (2008), pp. 2235-2247.

(21) Tamura, A., Okuyama, K., Takahashi, S. and Ohtsuka, M., Three-dimensional discrete -velocity BGK model for the incompressible Navier-Stokes equations, Computers and Fluids, Vol. 40, (2011), pp. 149-155.

(22) Tamura, A., Okuyama, K., Takahashi, S. and Ohtsuka, M., Development of numerical analysis method of flow-acoustic resonance in stub pipes of safety relief valves, Journal of Nuclear Science and Technology, Vol. 49, Nos. 7-8, (2012), pp.793-803.

(23) Chen, H., Chen, S. and Matthaeus, W. H., Recovery of the Navier-Stokes equations using a lattice gas Boltzmann method, Physical Review A, Vol. 45, (1992), pp. 5339-5342.

(24) Qian, Y. H., d'Humieres, D. and Lallemand, P. Lattice BGK models for Navier-Stokes equation, Europhysics Letters, Vol. 17, (1992), pp. 479-484.

(25) He, X. and Luo, L. S., Theory of the lattice Boltzmann method: From the Boltzmann equation to the lattice Boltzmann equation, Physical Review E, Vol. 56, (1997), pp. 6811-6817.

(26) d'Humieres, D. Bouzidi, M. and Lallemand, P., Thirteen-velocity three-dimensional lattice Boltzmann model, Physical Review E, Vol. 63, (2001), 066702-1-7.

(27) d'Humieres, D. Ginzburg, I., Krafczyk, M., Lallemand, P. and Luo, L. S., Multiple-relaxation-time lattice Boltzmann models in three dimensions, Philosophical Transactions of the Royal Society A, Vol. 360, (2002), pp. 437-451.

(28) Guo, Z. Zheng, C. and Shi, B., An extrapolation method for boundary conditions in lattice Boltzmann method, Physics of Fluids, Vol. 14, (2002), pp. 2007-2010.

(29) Chen, S. and Doolen, D., Lattice Boltzmann method for fluid flows, Annual Review of Fluid Mechanics, Vol. 30, (1998), pp. 329-364.

(30) Germano, M., Piomelli, U., Moin, P. and Cabot, W. H., A Dynamic subgrid-scale eddy viscosity model, Physics of Fluids, Vol. A3, No. 7, (1991), pp. 1760-1765. 